\title{
A Diffusion Tensor Imaging Study on the Phonology-related Pathways in Cantonese-mandarin Bilinguals.
}

\author{
Xiaoyu Xu \\ Sun Yat-Sen University \\ Yuying Jin \\ Sun Yat-Sen University \\ Ning Pan \\ Sun Yat-Sen University \\ Muqing Cao \\ Sun Yat-Sen University \\ Jin Jing \\ Sun Yat-Sen University \\ Jingwen $\mathrm{Ma}$ \\ Guangdong Province Maternal and Child Health Care Hospital \\ Xiaoxuan Fan \\ Wuhan Childrens Hospital: Wuhan Women and Children Medical Care Center \\ Si Tan \\ Sun Yat-Sen University \\ Xiaojing Song \\ Sun Yat-Sen University \\ Xiuhong Li ( $\square$ lixh@mail.sysu.edu.cn ) \\ Sun Yat-Sen University https://orcid.org/0000-0001-8084-9706
}

\section{Research Article}

Keywords: Cantonese-Mandarin bilinguals, diffusion tensor imaging, phonological processing, TBSS

Posted Date: December 22nd, 2021

DOI: https://doi.org/10.21203/rs.3.rs-1168282/v1

License: (c) This work is licensed under a Creative Commons Attribution 4.0 International License. Read Full License 


\section{Abstract}

Cantonese and Mandarin are logographic languages, and the phonology is the main difference between the two languages. It is unclear whether long-term experience of Cantonese-Mandarin bilingualism will shape different brain white matter structures of pathways related to phonological processing. 30 Cantonese-Mandarin bilinguals and 30 Mandarin monolinguals completed diffusion-weighted imaging (DWI) scans and phonological processing tasks. The tractography and TBSS were used to investigate the structural differences in the bilateral superior longitudinal fasciculus (SLF), inferior longitudinal fasciculus (ILF) and inferior fronto-occipital fasciculus (IFOF) between Cantonese-Mandarin bilinguals and Mandarin monolinguals. Post-hoc correlation analysis was conducted to investigate the relationship between the different structures with phonological processing skills. Compared to the Mandarin monolinguals, the Cantonese-Mandarin bilinguals had higher fractional anisotropy (FA) along the left ILF, higher mean diffusivity (MD) in the clusters along the temporoparietal segment of SLF (tSLF), as well as higher axial diffusivity (AD) in the right tSLF, IFOF, bilateral ILF. The mean AD of the different voxels in the right IFOF and the mean FA of the different voxels in the left ILF were positively correlated with the inverse efficiency score (IES) of the Cantonese auditory and Mandarin visual rhyming judgment tasks respectively within the bilingual group. Long-term experience of Cantonese-Mandarin bilinguals shape different brain white matter structures including right tSLF, IFOF, bilateral ILF. The bilinguals' white matter showed higher diffusivity, especially in the axonal direction, than the monolinguals. These changes were related to bilinguals' phonological processing.

\section{Introduction}

In the world, there are 55 countries with more than one official languages, and immeasurable regions with only one official language but also regional and local languages (www.uottawa.ca/clmc/). Understandably, countless people use more than one languages frequently for a long time. Long-term bilingual experience has an effect on multiple cognitive functions (see a review (Costa \& Sebastian-Galles, 2014)). The effect of bilingualism on phonological processing received much attention. Bilinguals rely on one brain to process two distinct phonologies. Functional imaging studies showed that the brain activation patterns during phonological processing of the native and second language (respectively L1 and L2) for bilinguals were different (see reviews (Liu \& Cao, 2016; Sulpizio, et al., 2020)), as well as the brain activation pattern during phonological processing of either language for bilinguals was different from that of the single language for monolingual (Cao, et al., 2013; Ma, et al., 2020; Parker Jones, et al., 2012). Functional changes are accompanied by anatomical changes (see reviews (Li, et al., 2014; Stein, et al., 2014)). White matter coordinates communications between different brain regions (Douglas, 2008) and interacts with the cortex function (Duffau, 2015). White matter has lifelong plasticity (Fields, 2015; Gibson, et al., 2014; McKenzie, et al., 2014) and is likely to be modulated by environment stimulus and behavioral experience, such as bilingualism (Li, et al., 2014). Therefore, it's reasonable to hypothesize that long-term bilingual experience can shape different white matter structures of phonological-related tracts.

Diffusion tensor imaging (DTI) technique allowed a detailed observation of white matter structure in vivo without invasion (Basser \& O"zarslan, 2014). Characteristics of white matter can be described by the DTI indices, such as fractional anisotropy (FA) and diffusivity measurements including mean, axial and radial diffusivities (MD, AD and RD respectively) (Soares, et al., 2013). According to the dual-stream model of language processing, the dorsal cortical circuit including the perisylvian language areas is demonstrated to be involved in processing the phonological information in written and spoken languages (Hickok \& Poeppel, 2007; Schlaggar \& McCandliss, 2007). Fiber dissection studies claimed that the superior longitudinal fasciculus (SLF) is the core fiber connecting the dorsal language cortical regions (Sarubbo et al., 2016). On the other hand, the ventral cortical circuit is generally considered to play an important role in semantic processing, while it also supports auditory comprehension during speech processing (Hickok \& Poeppel, 2007). The core tracts connecting the ventral stream consist of the inferior longitudinal fasciculus (ILF) and the inferior fronto-occipital 
fasciculus (IFOF) (Sarubbo, et al., 2016). These two tracts were also claimed to correlates the mapping from orthography to phonology (Mei, et al., 2015; Tomasino, et al., 2020).

Accumulating DTI evidence revealed that alphabetic-alphabetic bilinguals had different white matter structures from their monolingual peers, especially in the SLF, ILF and IFOF (Anderson, et al., 2018; Gold, et al., 2013; Kuhl, et al., 2016; Luk, et al., 2011; Singh et al., 2017; Pliatsikas, et al., 2015; Schlegel, et al., 2012). However, the reproducibility of these studies is low. It's considerable, because bilingualism is a multifaceted construct sensitive to age of L2 acquisition (AoA), L2 immersion time, language categories and etc. (Li, et al., 2014). Most of the current DTI studies on bilingualism recruited the first generation immigrants or local L2 learners as the bilingual samples, who generally learn L2 lately and use L2 for a short time (Cummine \& Boliek, 2013; Kuhl, et al., 2016; Pliatsikas, et al., 2015; Rossi, et al., 2017; Schlegel, et al., 2012). In the SLF, short-term late bilinguals usually demonstrated higher FA and lower RD than monolinguals (Pliatsikas, et al., 2015; Rossi, et al., 2017; Schlegel, et al., 2012). This is similar to the white matter characteristics of the subjects after L2 training (Hosoda, et al., 2013; Mamiya, et al., 2018). However, for bilinguals born in bilingual societies, they learn L2 at an early age, and the stimulus of the two languages are long-term and continuous. Only a few DTI studies on bilingualism recruited long-term bilinguals who learned L2 at an early age (Anderson, et al., 2018; Luk, et al., 2011; Singh et al., 2017). Anderson et al. (Anderson, et al., 2018) and Singh et al. (Singh et al., 2017) respectively recruited lifelong bilingual samples from Canada and Hindi, and matched them well with the monolingual samples. They both reported bilinguals had higher AD in the SLF than monolinguals, and Singh et al. also reported bilinguals had higher MD in the SLF than monolinguals. This is inconsistent with the results of the studies focusing on late short-term bilinguals (Pliatsikas, et al., 2015; Rossi, et al., 2017; Schlegel, et al., 2012). It's speculated that long-term immersion in a bilingual environment from an early age might induce different effects from relatively short-term L2 experience (Singh et al., 2017). In the world, there are countless regions with more than one language (www.uottawa.ca/clmc/), and nearly $66 \%$ of people are raised as bilingual speakers from childhood and use more than one language for a long time (Marian \& Shook, 2012). The white matter structural characteristics of such long-term bilinguals are of our interest.

In addition, rare studies focused on logographic bilinguals. Literature (Li, et al., 2014) indicated that the language typology could affect the changes in bilinguals' brain structure. Different from alphabetic languages, Chinese is a unique language best known for its logographic writing system (Deng, et al., 2013). Chinese characters have complex visualspatial configurations (Cao, et al., 2013). The orthography-to-phonology mapping rule is extremely opaque in Chinese, while it's relatively transparent in alphabetic languages (Cao, et al., 2017). In monolinguals, evidence from fMRI studies suggested that compared to alphabetic languages, mapping orthography to phonology in Chinese require extra involvement of cortex in the ventral stream (for details see the references (Bolger, et al., 2005; Sun, et al., 2011; Tan, et al., 2005)). For bilinguals, when processed as the L2 in the phonological tasks, compared to alphabetic language processing, Chinese processing also need greater activation in the ventral cortical regions similar to the regions required in Chinese processing for monolinguals (Cao, et al., 2017; Kim, et al., 2016). The ILF and IFOF are the two core tracts connecting the ventral cortex (Sarubbo, et al., 2016). Qi et al. (Qi, et al., 2015) found FA and RD of the right ILF could predict the Chinese achievement of native English speakers after short-term Chinese training. Besides, Cummine et al. (Cummine \& Boliek, 2013) observed Chinese-English bilinguals had different DTI indices in the bilateral IFOF and right ILF from English monolinguals. These two studies suggested that using Chinese and another alphabetic language together might be related to the white matter tracts in the ventral pathway. However, there has been no study focusing on the whiter matter characteristics of bilinguals using two kinds of logographic languages. In addition, none of the above two studies focused on long-term bilinguals who learn L2 at an early age. Thus, we aim to investigate how the long-term bilingual experience of two logographic languages may shape the white matter structure. We hypothesized that the white matter structure in the SLF, ILF and IFOF might exhibit an effect.

Mandarin and Cantonese are two major Chinese and both logographic languages. In Guangdong Province, China, nearly half of the population speak both Cantonese and Mandarin (Huiming \& Zhe, 2016). For Cantonese-Mandarin bilinguals, 
Cantonese is their native language (L1) mainly for daily communication and local medium, while Mandarin is the L2 for formal situations. As the official language, Mandarin is popularized nationwide throughout China. Mandarin is the teaching language in Guangdong, and children learn how to pronounce and write in Mandarin from primary school or even earlier. In terms of linguistic characteristics, Cantonese and Mandarin share the same set of characters and have similar grammatical structures (Tardif, et al., 2009). However, they shared the same pronunciation for only $21.5 \%$ of characters ( $\mathrm{Li}, 1990)$. Thus, although Cantonese is defined as a dialect according to socioeconomic factors, it is still deemed as an individual language in the region of psycholinguistics (Chen, et al., 2004; Tardif, et al., 2009). Evidence from behavioral studies suggested that Cantonese-Mandarin bilinguals performed different phonological processing skills compared to their Mandarin monolingual peers over a wide age span (Chen, et al., 2004; Li, et al., 2011). As reviewed above, the SLF is the most recognized tract involved in phonological processing, while the ventral stream including the ILF and IFOF also play a role in speech comprehension and orthography-to-phonology mapping. It's assumed that the white matter structure in SLF, ILF and IFOF might be different between Cantonese-Mandarin bilinguals and Mandarin monolinguals, and the different structure might have a relationship with phonological processing.

In the current study, we used the Tract-Based Spatial Statistics (TBSS) analysis to test our hypothesis. Tractography was conducted to create a binary mask of tracts of interests (TOI) for TBSS analysis to improve the anatomical accuracy and the power to detect significance (Hamalainen, et al., 2017). We measured the phonological processing skills and performed a post-hoc correlation between the phonological processing skills and the significant clusters revealed in the group-wise comparison. The post-hoc comparison could help us better understand the white matter plasticity induced by Cantonese-Mandarin phonological processing. The aims of the current study were: 1) to test whether the white matter structure in phonology-related pathways including SLF, ILF and IFOF is different between Cantonese-Mandarin bilinguals and Mandarin monolinguals. 2) to explore the relationship between the phonological processing skills and the significant clusters in the white matter structure.

\section{Methods}

\subsection{Participants}

31 Cantonese-Mandarin bilinguals and 30 native Mandarin monolinguals were recruited from Canton. All the subjects underwent diffusion-weighted imaging (DWI) and T1-weighted scans. One participant in the bilingual group was excluded because his DWI image was incomplete. Finally, we included 30 Cantonese-Mandarin bilinguals (6 males; mean age $=21.17 \mathrm{y}$, standard deviation $(S D)=1.97 \mathrm{y}$ ) and 30 Mandarin monolinguals (9males; mean age $=21.40 \mathrm{y}, \mathrm{SD}=2.03 \mathrm{y}$ ) in analysis. The bilinguals' second language (L2) acquisition age ranged from 3 to 7 years old. The proficiency of the languages of participants was evaluated referring to the Language and Social Background Questionnaire (LBSQ). LBSQ is a self-assessment tool and has been proven to be reliable and valid in diverse languages (Anderson, et al., 2018). The bilinguals in this study were highly proficient in speaking and understanding both languages (Table 1) and grew up in Guangdong province, a Cantonese-Mandarin bilingual society. The monolinguals were all born and grew up in Mandarin regions. Also, since English is a compulsory course in the Chinese education system, all participants in this study had English learning experiences. We use grades of College English Test Band 4 (CET4), a national English test in China for undergraduate and postgraduate students, to evaluate participants' English proficiency and included CET4 grades as a nuisance variable in the group-wise comparison. The participants' non-verbal intelligence quotient evaluated by Raven's Standard Progressive Matrices Test was matched in the two groups. 
Table 1

Demographic characteristics and phonological processing skills.

\begin{tabular}{|c|c|c|c|c|c|c|c|}
\hline & \multicolumn{2}{|c|}{ Bilinguals $(N=30)$} & \multicolumn{5}{|l|}{$\begin{array}{l}\text { Monolinguals } \\
(N=30)\end{array}$} \\
\hline & Mandarin & Cantonese & Mandarin & $\mathrm{P}$ & $\mathrm{P}_{1}$ & $\mathrm{P}_{2}$ & $\mathrm{P}_{3}$ \\
\hline Age ( $M \pm S D$, years) & $21.17 \pm 1.97$ & & $21.40 \pm 2.03$ & 0.653 & - & - & - \\
\hline Gender (female/male, $M$ ) & $24 / 6$ & & $21 / 9$ & 0.552 & - & - & - \\
\hline IQ score $(M \pm S D)$ & $122.40 \pm 11.8$ & & $124.33 \pm 14.16$ & 0.569 & - & - & - \\
\hline Acquisition age $(M \pm S D)$ & $4.53 \pm 1.33$ & $1.15 \pm 0.83$ & $1.73 \pm 1.13$ & - & $<0.001$ & $<0.001$ & 0.067 \\
\hline \multicolumn{8}{|l|}{ Socio-economic status $(\mathrm{N})$} \\
\hline $\begin{array}{l}\text { Education } \\
\text { (undergraduate/postgraduate) }\end{array}$ & $22 / 8$ & & $23 / 7$ & 0.766 & - & - & - \\
\hline $\begin{array}{l}\text { District of residence } \\
\text { (city/suburb/missing) }\end{array}$ & 19/10/1 & & $20 / 10 / 0$ & 0.926 & - & - & - \\
\hline Father's education & & & & 0.894 & - & - & - \\
\hline Junior high school or below & 13 & & 11 & & & & \\
\hline $\begin{array}{l}\text { Senior high school or } \\
\text { technical secondary school }\end{array}$ & 11 & & 12 & & & & \\
\hline College or above & 6 & & 7 & & & & \\
\hline Mother's education & & & & 0.364 & - & - & - \\
\hline Junior high school or below & 11 & & 13 & & & & \\
\hline $\begin{array}{l}\text { Senior high school or } \\
\text { technical secondary school }\end{array}$ & 16 & & 11 & & & & \\
\hline College or above & 3 & & 6 & & & & \\
\hline \multicolumn{8}{|l|}{ Language proficiency $(\mathrm{M} \pm \mathrm{SD})$} \\
\hline Speaking ${ }^{2}$ & $8.50 \pm 1.11$ & $9.43 \pm 0.77$ & $8.87 \pm 1.20$ & & 0.162 & 0.001 & 0.053 \\
\hline Writing 23 & $8.57 \pm 1.14$ & $5.73 \pm 2.13$ & $9.03 \pm 0.93$ & & 0.433 & $<0.001$ & $<0.001$ \\
\hline Reading ${ }^{23}$ & $8.87 \pm 0.97$ & $7.77 \pm 1.43$ & $8.77 \pm 1.17$ & & 0.479 & $<0.001$ & 0.003 \\
\hline comprehension & $8.83 \pm 1.12$ & $8.83 \pm 0.91$ & $8.77 \pm 1.28$ & & 0.785 & 0.941 & 0.926 \\
\hline CET4 grades ${ }^{*}$ & $557.00 \pm 45.9$ & & $517.78 \pm 60.69$ & 0.007 & - & - & - \\
\hline \multicolumn{8}{|c|}{ Phonological processing skills (M (SD)) } \\
\hline Visual rhyming task IES $\mathrm{S}^{23}$ & $6.74 \pm 1.93$ & $8.34 \pm 3.15$ & $5.69 \pm 2.29$ & & 0.017 & $<0.001$ & 0.008 \\
\hline Auditory rhyming task IES ${ }^{23}$ & $8.36 \pm 3.45$ & $10.14 \pm 4.25$ & $7.17 \pm 2.18$ & & 0.270 & $<0.001$ & 0.003 \\
\hline Digits span score ${ }^{2}$ & $31.27 \pm 3.87$ & $33.17 \pm 3.37$ & $32.27 \pm 4.43$ & & 0.277 & 0.005 & 0.380 \\
\hline RAN-digits RT $(m s)^{12}$ & $0.30 \pm 0.06$ & $0.28 \pm 0.04$ & $0.26 \pm 0.04$ & & 0.001 & 0.015 & 0.019 \\
\hline
\end{tabular}




\begin{tabular}{|c|c|c|c|c|c|c|}
\hline & \multicolumn{2}{|c|}{ Bilinguals $(N=30)$} & \multicolumn{4}{|l|}{$\begin{array}{l}\text { Monolinguals } \\
(N=30)\end{array}$} \\
\hline RAN-object RT (ms) & $0.58 \pm 0.09$ & $0.59 \pm 0.11$ & $0.57 \pm 0.08$ & 0.906 & 0.473 & 0.548 \\
\hline \multicolumn{7}{|c|}{$\begin{array}{l}\text { Note: } P_{1} \text { is the significance of the comparison between Cantonese-Mandarin bilinguals performing the tasks in } \\
\text { Mandarin }(\mathrm{CM}) \text { and Mandarin monolinguals performing the tasks in Mandarin }(\mathrm{MM}) . P_{2} \text { is the significance of the } \\
\text { comparison between } \mathrm{CM} \text { and Cantonese-Mandarin bilinguals performing the tasks in Cantonese }(\mathrm{CC}) . P_{3} \text { is the } \\
\text { significance of the comparison between the CC and MM. } a \text { was } 0.05 \text { for variables in only two groups and was } \\
\text { corrected by Bonferroni's correction for the comparisons of variables in all } 3 \text { groups }\left(a_{\text {corrected }}=0.05 / 3=0.017\right) \text {. } \\
\text { Superscripts were labeled for variables with } P \text { values below the thresholds, which were also in bold. }\end{array}$} \\
\hline
\end{tabular}

All participants were right-handed referring to the Edinburgh Handedness Inventory (Oldfield, 1971). The ones with learning disabilities, neurological diseases, psychiatric disorders, visual and hearing difficulties, attention deficit hyperactivity disorder, or contraindications of MRI were excluded. All participants signed written informed consent before participating. The Medical Ethics Committee, Sun Yat-sen University provided ethical approval for this study with the ethical approval number [L2016] No.036.

\subsection{Behavioral measures of phonological processing skills}

We chose the rhyming judgment task, rapid automatized naming task (RAN), and digit span test to assess the three aspects of phonological processing skills (Wagner \& Torgesen, 1987) respectively. For bilinguals, we performed the tasks in both Mandarin and Cantonese, while only in Mandarin for monolinguals.

\subsubsection{Rhyming judgment task}

The visual and auditory rhyming tasks were performed to test the phonological awareness of getting sound-based representations from the written and spoken words. In the visual task, the participants needed to transfer the orthography to phonology first, while they didn't in the auditory task. Both the rhyming tasks were displayed on Eprime2.0 based on previous studies on Chinese adults (Cao, et al., 2017). Each task consisted of 30 trials. The design of the tasks was displayed in Supplemental Figure 1. All the words used in the tasks consisted of one onset and one rime and the two paired words had the same tone. The participants were asked to judge whether the two paired words had the same rhyme. Characters used in the visual rhyming judgment tasks were the common words chosen from the Modern Chinese Dictionary and the Cantonese Dictionary respectively. The average reaction time (RT) of responses and the accuracy rate (AR) were recorded for either task. An integrated variable called inverse efficiency score (IES) was computed by dividing RT by AR (IES=RT/AR) (Bruyer \& Brysbaert, 2011) and used as the measurement of rhyming judgment task performance.

\subsubsection{Rapid automatized naming}

The classical RAN was used to assess the ability of phonological lexical retrieval (Siddaiah, et al., 2016). We used digits and objects as the materials. 40 symbols were printed on A4 paper and participants were asked to read them twice as fast and accurately as possible. The average RT (ms) of RAN was calculated by dividing the total RT (ms) by the mean number of correct reactions.

\subsubsection{Digit span test}

The "Digit Span" test from the Wechsler Adult Intelligence Scale-Revised Chinese version (WAIS-SR) was used to assess verbal working memory ability. The number of correct answers was recorded as the total score.

\subsection{MRI acquisition and analysis}




\subsubsection{MRI acquisition}

Diffusion-weighted images (DWI) were acquired by a 3.0 T Siemens Scanner (Siemens Healthcare, Erlangen. Germany) at the Huanan Normal University in Guangzhou. A single-shot spin-echo echoplanar imaging sequence was used with the following parameters: repetition time (TR) $10000 \mathrm{~ms}$, echo time (TE) $90 \mathrm{~ms}$, flip angle $90^{\circ}$, matrix size $=128 \times 128$, field of view $256 \mathrm{~mm}$, voxel size $2 \times 2 \times 2 \mathrm{~mm}^{3}$, b-value $1000 \mathrm{~s} / \mathrm{mm}^{2}$, number of average 1 , and GRAPPA factor 2 . Diffusion gradients in 64 non-collinear directions with one image of b-value of $0 \mathrm{~s} / \mathrm{mm}^{2}$ were collected.

T1-weighted 3D images were acquired using magnetization prepared rapid gradient-echo sequence with following parameters: $\mathrm{TR}=1900 \mathrm{~ms}, \mathrm{TE}=2.52 \mathrm{~ms}$, flip angle $=90^{\circ}$, matrix size $=256 \times 256$, field of view $=256 \mathrm{~mm}$, voxel size $1 \times 1 \times 1 \mathrm{~mm}^{3}$, number of average 1 .

\subsubsection{MRI preprocessing and tractography}

MRI preprocessing was performed using FSL (version 5.0.9) (FMRIB Software Library, https://fsl.fmrib.ox.ac.uk/fsl/). The procedures included visual inspection of quality control, eddy correction (Andersson \& Sotiropoulos, 2016), deletion of non-brain tissue (Smith, 2002), calculation of DTI to get FA, MD and RD maps (Behrens, et al., 2003). Additionally, DTI data quality was quantificationally evaluated according to the log file generated in eddy correction using trac-all qa tool (Yendiki, et al., 2014) in FreeSurfer. The mean of average translation $(\mathrm{mm})$ was $0.76 \pm 0.24$ for bilinguals and $0.78 \pm 0.21$ for monolinguals, while the mean of average rotation $\left(\times 10^{-2}\right.$ ) was $0.47 \pm 0.12$ for bilinguals and $0.46 \pm 0.10$ for monolinguals. The percentage of bad slices for both groups was 0 and the average signal drop-out score was 1 . No difference was found in DWI quality measures between the two groups.

Tractography was performed using diffusion toolkit and trackvis (http://trackvis.org), with the parameter: minimum FA threshold 0.2 , step length $0.5 \mathrm{~mm}$, maximum angle threshold $35^{\circ}$. To minimize the subjective variation of manual Regions of interest (ROI) definition, we used the semiautomatic method to define ROls according to Su et al. (Su, et al., 2018). We followed the acknowledged protocol to dissect ILF and IFOF (Catani \& Thiebaut de Schotten, 2008). Because there are multiple names of the SLF subdivisions, which confuse the readers, we followed the classification proposed by Nakajima et al. (Nakajima, et al., 2019) to divide the SLF to four parts: dorsal SLF, ventral SLF, AF and temporoparietal segment of SLF (tSLF). Since the dorsal and ventral SLF are difficult to be divided using DTI model (De Schotten, et al., 2011), and the functions of them are highly correlated (Budisavljevic, et al., 2017, Nakajima, et al., 2019), we reconstructed them together following the protocol proposed by Thiebaut et al. (De Schotten, et al., 2011) and used frontoparietal segment of SLF (fSLF) to referred the merged tract. The AF and tSLF were reconstructed following the protocols proposed by Catani et al. (Catani, et al., 2005).

\subsubsection{MRI data processing}

We performed tract-specific TBSS analysis to compare white matter structures of the TOI between the two groups. Every subject's binary mask of TOI was generated using the fslmaths tool in native space and registered to the MNI space. Then, we create an averaged binary mask of TOI by including the voxels belonged to $75 \%$ participants. The TBSS analysis was performed according to the standard pipeline supplied in FSL website (Smith, et al., 2006). The averaged binary mask was used in the permutation tests. Threshold-Free Cluster Enhancement (Smith \& Nichols, 2009) with 10,000 permutations was set. Family-wise error rate was corrected. The location of significant voxels in the tract-specific analysis was reported through the averaged binary masks of separate tracts of TOI.

To explore the group-wise difference outside the TOI and facilitate the comparison with previous studies, we also performed the whole-brain TBSS analysis. The mean FA map was skeletonized and the minimum threshold of 0.2 was set to create a mean white matter skeleton. The FA, MD, AD and RD values of all participants were projected to the 
skeleton in MNI space. The white matter skeleton was used as the mask in the permutation tests (Winkler, et al., 2014), setting 10000 permutations, controlling family-wise error (FWE) rate, with threshold-free cluster enhancement (Smith \& Nichols, 2009). The averaged binary masks of separate tracts of TOI were used to report the location of the significant voxels in the TOI, while JHU white-matter tractography atlas (Hua, et al., 2008) was used to report the location of the significant clusters outside the TOI.

The mean values of the different DWI parameters of each significant cluster was extracted using fslmeants tool for the post-hoc association analysis.

\subsection{Statistical analysis}

Statistical analysis was performed using R4.0.2. Comparison of demographic information and behavioral measures between the bilingual group and monolingual group was conducted by independent-samples $t$-tests, Mann-Whitney $U$ tests, or chi-square test. Wilcoxon paired-samples signed rank tests were performed for Cantonese and Mandarin proficiency and phonological processing skills comparison within the bilingual group. Uncorrected $P$-values were reported and compared with Bonferroni-corrected $a$.

For post-hoc subgroup correlation analysis, Pearson correlation analysis and Spearman rank correlation analysis were performed separately for variables in normality and non-normality. The variables for Spearman rank correlation were RT of the Mandarin RAN digit task, IES of the Cantonese auditory rhyming task, IES of the Cantonese visual rhyming task within the bilingual group, and none in the monolingual group. Concerning the family-wise error (FWE), a partial Bonferroni correction was performed according to the procedure published on the SISA website (https://www.quantitativeskills.com/sisa/calculations/bonhlp.htm). The corrected $a$ was 0.010 for the bilingual group, and 0.014 for the monolingual group. The correlation coefficients with an uncorrected $P$ were presented in the Table 3 , but only the correlations survived the FWE correction were showed in Fig. 3.

\section{Results}

\subsection{Phonological processing skills}

The performance of phonological processing skills was reported in Table 1. CC (Cantonese-Mandarin bilinguals performing the tasks in Cantonese) demonstrated higher IES than CM (Cantonese-Mandarin bilinguals performing the tasks in Mandarin) and MM (Mandarin monolinguals performing the tasks in Mandarin) in both visual and auditory rhyming judgment tasks $(P<0.017)$. The $\mathrm{CM}$ also showed higher IES than MM in the visual rhyming judgment task (CM: $6.74 \pm 1.93$ VS MM: $5.69 \pm 2.29, P=0.017)$. The higher the IES, the poorer the phonological awareness. Furthermore, $C C$ got a higher score in the digit span task than CM (CC: $33.17 \pm 3.37$ VS CM: $33.17 \pm 3.37, P=0.005)$. CM performed slower than $\mathrm{CC}$ and $\mathrm{MM}$, in the digit RAN task (CM: $0.30 \pm 0.06$ VS CC: $0.28 \pm 0.04$ and MM: $\left.0.26 \pm 0.04, P_{C M-C C}=0.001, P_{C M-M M}=0.015\right)$.

\subsection{The results of tractography}

The tractography of fSLF, tSLF, ILF and IFOF succeeded in all the subjects, but AF known not to be traceable in all individuals (Catani, et al., 2007; Vanderauwera, et al., 2017) was failed to be reconstructed in a few subjects. The right AF couldn't be reconstructed in 13 bilinguals and 3 monolinguals, while the left AF couldn't be reconstructed in 5 bilinguals and 2 monolinguals.

\subsection{Comparison of white matter structure between bilinguals and monolinguals.}

The tract-specific TBSS analysis revealed that compared to the Mandarin monolinguals, Cantonese-Mandarin bilinguals had higher MD in the right ISLF, higher FA in the left ILF and higher AD in the left ILF and right IFOF after controlling the 
English proficiency and average translation ( $p<0.05$, FWE corrected). The results are represented in Fig. 1, and the peak coordinates and number of the differential voxels distributed in each tract are reported in Table 2. Monolinguals didn't show higher FA, MD or AD than bilinguals in any voxel. In addition, RD didn't yield significance in the tract-specific TBSS analysis.

Table 2

Results of the comparison of white matter structure between bilinguals and monolinguals.

\begin{tabular}{|c|c|c|c|c|c|}
\hline $\begin{array}{l}\text { Level of } \\
\text { analysis }\end{array}$ & White matter Tract & DTI parameter & Voxels & $\begin{array}{l}\text { Peak } \\
\text { coordinate }\end{array}$ & $P_{\min }$ \\
\hline \multirow[t]{4}{*}{ Tract-specific } & tSLF (R) & $\begin{array}{l}\text { MD } \\
\text { (Bilingual>Monolingual) }\end{array}$ & 39 & $(43,-48,8)$ & 0.011 \\
\hline & $\operatorname{ILF}(\mathrm{L})$ & FA (Bilingual>Monolingual) & 24 & $(-40,-45,-5)$ & 0.018 \\
\hline & $\operatorname{ILF}(\mathrm{L})$ & AD (Bilingual>Monolingual) & 75 & $(-41,-43,-7)$ & 0.005 \\
\hline & IFOF(R) & AD (Bilingual>Monolingual) & 10 & $(34,-57,0)$ & 0.032 \\
\hline \multirow[t]{3}{*}{ Whole-brain } & tSLF (R) & $\begin{array}{l}\text { MD } \\
\text { (Bilingual>Monolingual) }\end{array}$ & 96 & $(43,-48,8)$ & 0.046 \\
\hline & CST (L) & $\begin{array}{l}\text { MD } \\
\text { (Bilingual>Monolingual) }\end{array}$ & 27 & $(-23,-34,43)$ & 0.049 \\
\hline & FMa & $\begin{array}{l}\text { MD } \\
\text { (Bilingual>Monolingual) }\end{array}$ & 19 & $(2,-42,11)$ & 0.049 \\
\hline & $\mathrm{tSLF}(\mathrm{R})$ & AD (Bilingual>Monolingual) & 133 & $(40,-45,19)$ & 0.017 \\
\hline & IFOF(R) & AD (Bilingual>Monolingual) & 369 & $(40,-41,-4)$ & 0.014 \\
\hline & $\operatorname{ILF}(L)$ & AD (Bilingual>Monolingual) & 28 & $(-41,-43,-7)$ & 0.046 \\
\hline & $\operatorname{ILF}(\mathrm{R})$ & AD (Bilingual>Monolingual) & 142 & $(49,-38,-11)$ & 0.012 \\
\hline & $\operatorname{ATR}(\mathrm{L})$ & AD (Bilingual>Monolingual) & 28 & $(-21,16,18)$ & 0.047 \\
\hline & $\operatorname{ATR}(\mathrm{R})$ & AD (Bilingual>Monolingual) & 94 & $(22,20,8)$ & 0.04 \\
\hline & $\operatorname{CST}(\mathrm{R})$ & AD (Bilingual>Monolingual) & 78 & $(27,-21,13)$ & 0.013 \\
\hline & $\begin{array}{l}\text { Hippocampal Cingulum } \\
\text { (R) }\end{array}$ & AD (Bilingual>Monolingual) & 79 & $(22,-42,-2)$ & 0.018 \\
\hline & FMa & AD (Bilingual>Monolingual) & 226 & $(-15,-41,13)$ & 0.02 \\
\hline & FMi & AD (Bilingual>Monolingual) & 91 & $(14,27,17)$ & 0.039 \\
\hline \multicolumn{6}{|c|}{$\begin{array}{l}\text { Note: CET4 grades and average translation (mm) were controlled, with FWE corrected and a lowest threshold of } 10 \\
\text { voxels for each cluster. Abbreviation list: temporoparietal segment of superior longitudinal fasciculus (tSLF), inferior } \\
\text { longitudinal fasciculus (ILF), inferior fronto-occipital fasciculus (IFOF), corticospinal tract (CST), forceps minor (FMi), } \\
\text { forceps major (FMa), anterior thalamic radiation (ATR), left (L), right (R). }\end{array}$} \\
\hline
\end{tabular}

The whole-brain TBSS analysis revealed higher MD in right tSLF, forceps major (FMa), and left corticospinal tract (CST), and higher AD in the right tSLF, IFOF, ILF, CST, hippocampal cingulum, bilateral anterior thalamic radiation (ATR), forceps minor (FMi) and FMa for Cantonese-Mandarin bilinguals controlling for CET4 grades and average translation $(p<0.05$, FWE corrected). The results are represented in Fig. 2, while the number and peak coordinates of the differential voxels distributed in each tract are reported in Table 2. Monolinguals didn't show higher MD or AD than bilinguals in any voxel. In addition, FA and RD didn't yield significance in the whole-brain TBSS analysis. 


\subsection{The results of post-hoc correlation analysis.}

As shown in Fig. 3, the mean FA of the different voxels in the left ILF was positively correlated with mandarin visual rhyming judgment task IES within the bilingual group $(r=0.498, P=0.006)$. The mean $A D$ of the different voxels in the right IFOF was positively correlated with the IES of Cantonese auditory rhyming judgment task within the bilingual group $(r=0.582, P=0.001)$. In addition, the mean MD of the cluster in the left CST was positively correlated with mandarin auditory rhyming task IES within the bilingual group $(r=0.564, P=0.001)$. The mean AD of the significant voxels in the right hippocampal cingulum was negatively correlated with the IES of the Mandarin auditory rhyming judgment task $(r=-0.520, P=0.005)$ within the monolingual group. All the above correlations survived the multiple correction. The detailed correlation coefficients and $P$ values of all the behavioral measures were provided in Supplemental Table1.

\section{Discussion}

We combined tractography and TBSS to investigate the difference in white matter structures along the major phonologyrelated pathways between Cantonese-Mandarin bilinguals and Mandarin monolinguals. The results support our hypothesis. We observed that compared to monolinguals, bilinguals had higher MD and AD along the right tSLF, and higher AD along the bilateral ILF and right IFOF, as well as higher FA along the left ILF. The difference pattern of DTI indices between Cantonese-Mandarin bilinguals and Mandarin monolinguals is close to that in previous studies on longterm alphabetic-alphabetic bilinguals (Anderson, et al., 2018; Singh et al., 2017), but not exactly the same. The inconsistency might be related to the specific characteristics of Cantonese and Mandarin. Furthermore, there were a significant relationship between the Mandarin phonological awareness and the mean DTI indices of the different voxels along the ventral tracts, CST and hippocampal cingulum.

\subsection{Differences in phonological processing skills}

For the skills of the two languages of the bilinguals, we observed that Cantonese-Mandarin bilinguals had worse Cantonese phonological awareness than Mandarin, in both visual and auditory rhyming tasks. It is reasonable, considering that Cantonese-Mandarin bilinguals in mainland China only accept pinyin instruction for Mandarin but not Cantonese in school. Pinyin is the phonological coding system used in China. As both Mandarin and Cantonese are morphosyllabic, which are not divisible at the phoneme, pinyin instruction can greatly facilitates the phonological awareness among the Chinese (Shu, et al., 2008). The deficiency of pinyin instruction for Cantonese may lead to the poorer Cantonese phonological awareness of the bilinguals. In addition, Cantonese-Mandarin bilinguals had better digit span and RAN performance in Cantonese than Mandarin. This phenomenon is consistent with previous evidence that bilinguals have better abilities of working memory (Grundy \& Timmer, 2016) and phonological lexical retrieval (Pennino, 2010; Yeung, 2016) in their native languages than their $L 2$.

As to the differences between bilinguals and monolingual, we observed that Cantonese-Mandarin bilinguals performed worse in the Mandarin visual rhyming judgment task and digit RAN task than Mandarin monolinguals. The results reflect the negative language transfer from Cantonese to Mandarin. The differences will be discussed combined with the neuroimaging results below. The lower speed of naming is probably related to the interference from non-target language when bilinguals are facing language choosing (Jian, et al., 2011).

\subsection{Dorsal white matter tracts}

First, we found that Cantonese-Mandarin bilinguals had higher MD and AD in the right tSLF than Mandarin monolinguals. MD measures the average diffusivity of water molecules across all directions, namely isotropy, and higher MD reflects more free water diffusion in white matter (Soares, et al., 2013). Literature showed that higher MD can accompany increased axonal caliber, more fiber crossings, looser packing density, fewer synapses or glial cells (Sagi, et 
al., 2012), or even an increase in tissue water content, such as increased cerebral blood flow (Jin \& Kim, 2008). AD refers to the diffusivity of water molecules parallel to fiber tracts. Higher AD was found to be associated to more axons, increased axonal caliber, looser packing density or more coherent orientation of axons, but not to be sensitive to myelin changes (Solowij, et al., 2017). A possible inference of our finding is that compared to Mandarin monolinguals, Cantonese-Mandarin bilinguals have higher axonal caliber or looser packing density in the right tSLF, considering the common microstructure properties the two DTI indices reflect.

Different from our finding, some intervention studies observed increased FA in the right SLF for L2 learners (Hosoda, et al., 2013, Mamiya, et al., 2016). For example, Hosoda et al. (Hosoda, et al., 2013) found that FA in the right SLF would increase after 16 weeks of English training for Japanese speakers. Mamiya et al. (Mamiya, et al., 2016) also found that FA in the right SLF was positively correlated with the number of training days in English immersion program for Chinese speakers. Animal models showed that the increase of FA was associated with learning (Gibson, et al., 2014). Hosoda and Mamiya guessed that the increase of FA might be associated with increased brain myelination. However, we believed that our results are less likely to reflect remodeling of myelin, because myelination is not a necessary condition for MD and AD changes (Solowij, et al., 2017). Furthermore, we found that there was no significant difference in FA and RD in the right SLF between the two groups. FA and RD are sensitive to myelin changes (Basser \& Pierpaoli, 2011; Song, et al., 2002). The $L 2$ training in both the intervention studies was short-term, whereas the Cantonese-Mandarin bilingual adults in our study use L2 regularly since an average age of 4.53 (range from 3 to 7). Evidence from motor training suggested that short-term intervention increased FA (Scholz, et al., 2009), while compared with short-term intervention, long-term training would induce increased diffusivity and reduced fiber coherence (Giacosa, et al., 2019). Accordingly, we speculated that the effect of long-term use of L2 on white matter might also be different from short-term training. Evidence from cross-sectional studies might supported our speculation. Consistent with our finding, Singh et al. (2017) reported that Hindi-English bilinguals had higher MD in the bilateral SLF and higher AD in the right SLF than Italian monolinguals. The Hindi-English bilinguals also lived in bilingual society and started to use L2 frequently since an average age of 5 . They suggested that lifelong bilingual language experience would generate greater isotropic diffusivity.

In addition, Anderson et al. (2018) also observed higher AD in the SLF for lifelong bilinguals in Canada compared to monolinguals after carefully matching between groups in age and general cognitive abilities. However, Anderson et al. didn't observe any difference in MD between the groups. The participants in Anderson's study were elders, while the participants in both Singh's and our studies were young adults. The developmental trajectories of white matter were different between bilinguals and monolinguals from early childhood to young adulthood (Pliatsikas, et al., 2020). It is possible that the developmental trajectories of white matter of bilinguals and monolinguals are also different from young adulthood to old age. We speculate that the group-wise difference in MD might decline gradually with age, but the difference in $A D$ would remain. To test this speculation, we performed a correlation of mean MD and AD of the different voxels in TSLF with age within the subgroups, and found a significant positive correlation between mean MD and age in monolinguals $(r=0.430, p=0.018)$, as well as a marginally significant negative correlation between mean MD and age in bilinguals ( $r=-0.321, p=0.084)$. Nevertheless, no significant correlation was found between mean AD and age $(p>0.10)$. According to the correlations, with age increase, the gap of MD between groups will gradually decrease, but the gap of AD won't change, which supported our speculation. Evidence from larger samples with larger age span will be needed in the future. There are also some cross-sectional studies reported contradictory findings in the SLF with our study. Luk et al. (2011) also recruited elder lifelong bilinguals with frequent L2 use, but they reported increased FA in the bilateral SLF. However, according to Anderson et al. (2018), the matching between the groups in Luk's study might not be enough considering the high popularity of preclinical dementia in elders. In addition, Pliatsikas et al. (2015) and Kuhl et al. (2016) both reported differences in FA in the bilateral SLF between bilinguals and monolinguals. Most of the bilinguals in the two studies started to immerse themselves in L2 environment in adulthood. The changes of FA might be related to the later exposure to L2 environment in adulthood. Overall, the pattern of increased MD and AD in the SLF for CantoneseMandarin bilinguals might have relationship with the long-term frequent use of two languages from an early age.

Page $11 / 25$ 
Furthermore, we noticed that the specific location in the SLF of difference reported in some studies differed from our findings. Few previous studies on bilingualism reported the specific subdivisions of SLF, but most of these studies not only reported differences in the right SLF but also the left SLF (Kuhl, et al., 2016; Luk, et al., 2011; Singh et al., 2017; Pliatsikas, et al., 2015), and Anderson et al. (2018) even only reported differences in the left SLF. We speculated that the inconsistency might be related to the different categories of languages used among those studies. The participants in previous studies were all alphabetic-alphabetic bilinguals, while in our study, the participants were logographiclogographic bilinguals. Unlike the prominent role of the left SLF in phonological processing, the right SLF was related to visuospatial functions (Nakajima, et al., 2019). The right tSLF connects the parietal lobe to the temporal lobe, and participants in visuospatial perception (Vaessen, et al., 2016). Besides, the right superior temporal gyrus that right tSLF connected is sensitive to tonal perception (Liang \& Du, 2018). Characters of logographic languages have complex spatial structures, and character processing in logographic languages demands more involvement of brain regions related to visuospatial processing especially in the right hemisphere than alphabetic languages (Bolger, et al., 2005; Tan, et al., 2005). In addition, both Cantonese and Mandarin are tonal languages. Using two tonal logographic languages might associate with white matter structural changes in the right tSLF. Furthermore, the left SLF was found to contribute to reading in English more than Chinese (Zhang, et al., 2014). Accordingly, we guess the effect of logographic-logographic bilingualism on the left SLF might not be distinct enough to be observed in relatively limited samples. No significant correlations were found between the mean different DTI metrics in the right tSLF and the phonological processing skills we measured. As mentioned above, the right tSLF was related to tonal and visuospatial perception, but our behavioral measures didn't cover these two ability, which might explain the absence of significant correlations. In addition, we should note that the post-hoc correlation analysis was performed in a relatively small sample with a strict multiple correction, and the absence of significance might also have a relationship with the limited statistic power.

\subsection{Ventral white matter tracts}

We also found that Cantonese-Mandarin bilinguals exhibited higher AD and FA in the left ILF, and higher AD in the right ILF and IFOF. Higher FA is accompanied by more myelination, more axons, higher axonal packing density, or less fiber crossing (Basser \& O"zarslan, 2014). The higher AD and FA in the left ILF in the bilinguals might indicate that CantoneseMandarin bilinguals have more axons, increased myelin or less fiber mixture. The higher AD in the right ILF and IFOF might indicate more axons, increased axonal caliber, looser packing density or more coherent orientation of axons (Solowij, et al., 2017). Different form the dorsal tracts, there was no difference in MD in the ventral tracts between the bilinguals and monolinguals. Consistent with our study, none of DTI studies on lifelong bilinguals observed a difference of MD in the ventral tracts (Anderson, et al., 2018; Luk, et al., 2011; Singh et al., 2017). Among these studies, Luk et al. (2011) and Anderson et al. (2018) respectively reported increased FA and AD in the bilateral ventral tracts including ILF and IFOF for bilingual elders compared to monolingual elders. Both Luk and Anderson suggested that the increased FA and $A D$ reflected enhanced white matter integrity in bilingual elders, and this neural adaption could serve as a buffer against the neuroatrophy of aging. Different from them, Singh et al. (2017) observed decreased FA and increased RD in the right ILF as well as decreased FA in the right IFOF for bilingual young adults compared to their monolingual peers. Singh et al. suggested that lifelong bilingual language experience would generate greater isotropic diffusivity in young adults. Among these studies, the association between lifelong bilinguals' experience and ventral white matter tracts was different because of different age. Interesting, the participants in the current study were also young adults, but the results were more consistent with that in the elder (Anderson, et al., 2018; Luk, et al., 2011), which showed that in addition to age, there are other factors that affect the relationship between lifelong bilingual experience and ventral white matter tracts.

Importantly, consistent with our hypothesis, we found the differences in the ventral tracts were related to phonological processing skills. First, we found that the mean FA and AD of the significant voxels in the left ILF was positively correlated with the IES of the Mandarin visual rhyming judgment task within the bilingual group (FA: $r=0.498, P=0.006$; AD: $r=0.454, P=0.013$ not survive the multiple correction), but not within the monolingual group. The correlations here

Page 12/25 
suggested that the increased FA and AD in the left ILF might have an association with the Mandarin phonological processing for Cantonese-Mandarin bilinguals. The left ILF is the crucial subcortical structure in the ventral stream. The ILF arises in occipital lobe, and projects to lateral and medial anterior temporal regions (Vandermosten, et al., 2012). Evidence from both alphabetic and logographic languages showed the bilateral ILF is involved in semantic processing, lexical retrieval, orthography perception and orthography-phonology conversion (Herbet, et al., 2018; Vandermosten, et al., 2012; Wang, et al., 2020). To complete the visual rhyming judgment tasks, subjects need to map orthography to phonology first and then decode the phonology (McPherson, et al., 1997). Our results might reflect a relationship between the left ILF and the ability of mapping orthography to phonology. Cantonese and Mandarin use the same set of written system, in which $70 \%$ of characters share the same orthography and meaning but not phonology in both languages, namely cognates (TÊ WANG, 1960). The activation of semantic representations was found to facilitate the phonological accessing of cognates in L2 (Friesen, et al., 2014). The left ILF plays an important role in visual semantic processing (Shin, et al., 2019). In addition, for bilinguals who use two languages with the same orthography but different phonological associations, they are more likely to recruit the ventral stream than dorsal stream in the orthographyphonology conversion in both L1 and L2 (Nosarti, et al., 2010). The correlation between the difference in the left ILF and Mandarin visual rhyming judgment task within the bilingual group might also have a relationship with the specific linguistic characteristics of Cantonese and Mandarin.

Second, we also found that a positive correlation between the mean AD of the different voxels in the right IFOF and the IES of Cantonese auditory rhyming judgment task within the bilingual group $(r=0.582, P=0.001)$. In addition, we noticed the mean $A D$ was also correlated with IES of Mandarin auditory rhyming judgment task within the bilingual group $(r=0.420, P=0.023)$, though this correlation didn't survive the multiple correction. The IFOF projects from the ventral occipital lobe to the inferior frontal gyrus (Vandermosten, et al., 2012). As a core tract in the ventral stream, the IFOF also participate in semantic processing (Almairac, et al., 2015; Mirman, et al., 2015; Vassal, et al., 2010) and orthographic processing (Vandermosten, et al., 2012). In addition, because the IFOF connect the frontal lobe, it also plays a role in the phonological processing in spoken language (Lebel, et al., 2013; Li, et al., 2017) and executive function (Perez-Iglesias, et al., 2010). We speculated that in order to process two proficient languages in daily oral communication, compared to Mandarin monolinguals, Cantonese-Mandarin bilinguals need have stronger ability of auditory phonological processing to process the target language and stronger executive function to inhibit non-target language (Green \& Abutalebi, 2013).

Finally, the current study showed that the $p$ value $(P=0.07)$ of the correlation between the mean AD in the right ILF and the IES of Mandarin auditory rhyming task within the bilingual group is marginal (corrected $a=0.01$ ). Qi et al. (2015) found that FA of the right ILF could predict Chinese orthographic and phonological skills of English native speakers after they learned Chinese. We speculated that there might be a potential relationship between the difference in the right ILF and auditory phonological processing within the bilingual group, but the effect might be obscured in the relatively limited sample.

\subsection{Differences in other tracts}

The current study also indicated that compared to Mandarin monolinguals, Cantonese-Mandarin bilinguals had higher $A D$ in the bilateral ATR, right CST, hippocampal cingulum, FMi and FMa, and higher MD in the left CST and FMa. The results might suggest that compared to processing a single language, processing two languages is more complex and need the involvement of wider brain networks, such as memory, motor and executive functional brain networks.

First, the ATR was related to the ability of executive function (Mamiya, et al., 2018; Smolker, et al., 2018). The FMi and FMa contain the fibers in the corpus callosum connecting bilateral frontal lobe and bilateral occipital lobe respectively (Goldstein, et al., 2020). Both the FMi and FMa take part in the interhemispheric communication, and the FMi was also implicated in executive function (Just, et al., 2007; Mamiya, et al., 2018). Previous studies repeatedly reported differences in the ATR, FMi and FMa between bilinguals and monolinguals (Anderson, et al., 2018; Cummine \& Boliek, 2013; Kuhl, et

Page $13 / 25$ 
al., 2016; Singh et al., 2017; Pliatsikas, et al., 2015; Rossi, et al., 2017). For example, Singh et al. (2017) observed that compared to monolinguals, long-term bilinguals had higher AD in the right ATR and FMi. Anderson et al. (2018) reported that compared to the well-matched monolinguals, lifelong bilinguals had higher AD in the FMa and bilateral anterior corona radiation which is a segment of ATR (Jang \& Yeo, 2014). It was well known that the bilinguals required brain networks of executive function to inhibit the non-target language so that the target language could be processed appropriately (Kroll, et al., 2014; Kroll, et al., 2015). It is reasonable that long-term bilingual experience shapes the brain white matter underlying executive function. Besides, compared to monolinguals whose dominant language regions were located in the left hemisphere, bilinguals recruited more regions distributed in bilateral hemispheres for processing both languages (Hull \& Vaid, 2007). Therefore, bilinguals might also have different pattern of interhemispheric communication compared to monolinguals.

Second, the CST originates in several regions of cerebral cortex including the motor areas, supplementary motor areas, premotor areas and primary somatosensory cortex and terminates on the spinal cord, controlling movements of the limbs and trunk (Welniarz, et al., 2017). In language, the CST, especially the left one, participates in articulation movement (Connally, et al., 2014; Farinella-Bocian, et al., 2007; Zhang, et al., 2014) and speech perception (D'Ausilio, et al., 2011). The right CST was also reported to play a role in articulation movement (Zhang, et al., 2014), but less frequently than the left one (Connally, et al., 2014; Farinella-Bocian, et al., 2007). The hippocampal cingulum is the hippocampal segment of the cingulum, and play an important role in long-term memory consolidation and retrieval (Bubb, et al., 2018). Consistent with our study, Kuhl et al. (2016) reported that compared to monolinguals, bilinguals had higher $\mathrm{AD}, \mathrm{RD}$ and $\mathrm{MD}$ in the bilateral CST and hippocampal cingulum.

Previous studies suggested that higher AD in bilinguals might reflect increased white matter integrity (Anderson, et al., 2018), and lifelong bilingual experience would increase diffusivity of white matter (Singh et al., 2017). MD represents the mean diffusion freedom of water molecules and is positively affected by AD (Basser \& Pierpaoli, 2011). Our results indicated that long-term Cantonese-Mandarin experience would increase diffusivity of white matter on executive function, long-term memory consolidation and retrieval, and articulation movement. Our resting-state fMRI study on whole-brain functional network between the Cantonese-Mandarin bilinguals and Mandarin monolinguals also supported that the bilinguals increased connectivity in subnetwork related to executive function, memory retrieval, and supplementary motor areas (Fan, et al., 2021). We observed that the mean MD of the significant voxels in the left CST was positively related to the IES of the Mandarin $(r=0.564, P=0.001)$ and Cantonese auditory rhyming judgment tasks $(r=0.438, P=0.017, a=0.010)$ only within the bilingual group. We also observed that the mean AD of the significant voxels in the right hippocampal cingulum was negatively correlated with the IES of the Mandarin auditory rhyming judgment task ( $r=-0.520, P=0.005)$ only within the monolingual group. The results indicated that bilinguals might need stronger articulation movement and memory retrieval to take part in the auditory phonological processing, thus increase diffusivity of CST and hippocampal cingulum.

\subsection{Limitations}

There are some limitations in this study. First, this study was a cross-sectionals which limited to explain the causal relationship between structural changes in white matter and long-term logographic-logographic bilingual experience. Second, the sample size was not large enough, which may affect the statistics effectiveness of the results. However, our sample size is still larger than most of the previous MRI studies on bilingualism (Cummine \& Boliek, 2013; Gold, et al., 2013; Kuhl, et al., 2016; Luk, et al., 2011; Singh et al., 2017; Pliatsikas, et al., 2015). Third, English is a compulsory course in mainland China, so all participants have English experience. To minimize the confounding, we controlled their English proficiency estimated by the CET4 grades in the group-wise comparison.

\section{Conclusion}

Page $14 / 25$ 
In conclusion, compared to Mandarin monolinguals, Cantonese-Mandarin bilinguals have different structures in the major phonology-related pathways including right tSLF, IFOF, bilateral ILF. The bilinguals' white matter showed higher diffusivity, especially in the axonal direction, than the monolinguals. The specific difference pattern of DTI indices in the dorsal stream may reflect the neuroplasticity related to long-term bilingual experience. As to the ventral tracts, they are not traditionally considered to participate in phonological processing. However, we found the differences in ventral white matter were related to phonological processing in Cantonese-Mandarin bilinguals. Our study confirmed the association between Cantonese-Mandarin bilingual experience and structural adaption in the ventral white matter tracts, and also the relationship between the structural adaption here and logographic language phonological processing skills in the bilinguals. Our study first provided evidence of white matter characteristics of bilinguals using two kinds of logographic languages.

\section{Declarations}

\section{Acknowledgments}

We thank all the participants for their participation in the study and the Brain Imaging Center of Institute for Brain Research and Rehabilitation in South China Normal University for their support in the data collection.

\section{Funding}

This work was supported by Key Realm R\&D Program of Guangdong Province [grant number 2019B030335001]; Guangdong Basic and Applied Basic Research Foundation [grant number 2021A1515011757]; and the National Natural Science Foundation of China [grant number 81673197].

\section{Competing Interests}

The authors have no relevant financial or non-financial interests to disclose.

\section{Author Contributions}

All authors contributed to the study conception and design. Material preparation, data collection were performed by Xiaoyu Xu, Xiaoxuan Fan, Si Tan, Xiaojing Song. Data analysis were performed by Xiaoyu Xu, Yuying Jin. Data Curation was performed by Ning Pan and Muqing Cao. Negotiation with the unit who helped us collect data was performed by Jin Jing. Supervision and funding acquisition were performed by Xiuhong Li. The first draft of the manuscript was written by Xiaoyu Xu and all authors commented on previous versions of the manuscript. All authors read and approved the final manuscript.

\section{Data Availability}

The datasets generated during and/or analysed during the current study are not publicly available due to individual privacy contained, but are available from the corresponding author on reasonable request. The individual private information will be deleted before sharing.

\section{Ethics approval}


This study was performed in line with the principles of the Declaration of Helsinki. Approval was granted by the Ethics Committee of Sun Yat-Sen University ([L2016] No.036).

\section{Consent to participate}

Informed consent was obtained from all individual participants included in the study.

\section{References}

1. Almairac, F, G Herbet, S Moritz-Gasser, N M de Champfleur and H Duffau (2015) The left inferior fronto-occipital fasciculus subserves language semantics: a multilevel lesion study. Brain Struct Funct 4, 1983-95 doi: $10.1007 / \mathrm{s} 00429-014-0773-1$

2. Anderson, J A E, J G Grundy, J De Frutos, R M Barker, C Grady and E Bialystok (2018) Effects of bilingualism on white matter integrity in older adults. Neuroimage 143-150 doi: 10.1016/j.neuroimage.2017.11.038

3. Anderson, J A E, L Mak, A Keyvani Chahi and E Bialystok (2018) The language and social background questionnaire: Assessing degree of bilingualism in a diverse population. Behav Res Methods 1, 250-263 doi: 10.3758/s13428-0170867-9

4. Andersson, J L R and S N Sotiropoulos (2016) An integrated approach to correction for off-resonance effects and subject movement in diffusion MR imaging. Neuroimage 1063-1078 doi: 10.1016/j.neuroimage.2015.10.019

5. Basser, P J and E O“zarslan (2014) Introduction to Diffusion MR.San Diego: Academic Press, pp. 3-9.

6. Basser, P J and C Pierpaoli (2011) Microstructural and physiological features of tissues elucidated by quantitativediffusion-tensor MRI. 1996. J Magn Reson 2, 560-70 doi: 10.1016/j.jmr.2011.09.022

7. Behrens, T E, M W Woolrich, M Jenkinson, H Johansen-Berg, R G Nunes, S Clare, P M Matthews, J M Brady and S M Smith (2003) Characterization and propagation of uncertainty in diffusion-weighted MR imaging. Magn Reson Med 5, 1077-88 doi: 10.1002/mrm.10609

8. Bolger, D J, C A Perfetti and W Schneider (2005) Cross-cultural effect on the brain revisited: universal structures plus writing system variation. Hum Brain Mapp 1, 92-104 doi: 10.1002/hbm.20124

9. Bruyer, R and M Brysbaert (2011) Combining speed and accuracy in cognitive psychology: Is the inverse efficiency score (IES) a better dependent variable than the mean reaction time (RT) and the percentage of errors (PE)?

Psychologica Belgica 1, 5-13 doi,

10. Bubb, E J, C Metzler-Baddeley and J P Aggleton (2018) The cingulum bundle: Anatomy, function, and dysfunction. Neurosci Biobehav Rev 104-127 doi: 10.1016/j.neubiorev.2018.05.008

11. Budisavljevic, S, F Dell'Acqua, D Zanatto, C Begliomini, D Miotto, R Motta and U Castiello (2017) Asymmetry and Structure of the Fronto-Parietal Networks Underlie Visuomotor Processing in Humans. Cereb Cortex 2, 1532-1544 doi: $10.1093 /$ cercor/bhv348

12. Cao, F, B L Sussman, V Rios, X Yan, Z Wang, G J Spray and R M Mack (2017) Different mechanisms in learning different second languages: Evidence from English speakers learning Chinese and Spanish. Neuroimage 284-295 doi: 10.1016/j.neuroimage.2017.01.042

13. Cao, F, R Tao, L Liu, C A Perfetti and J R Booth (2013) High proficiency in a second language is characterized by greater involvement of the first language network: evidence from Chinese learners of English. J Cogn Neurosci 10, 1649-63 doi: 10.1162/jocn_a_00414

14. Cao, F, M Vu, D H Chan, J M Lawrence, L N Harris, Q Guan, Y Xu and C A Perfetti (2013) Writing affects the brain network of reading in Chinese: a functional magnetic resonance imaging study. Hum Brain Mapp 7, 1670-84 doi: 10.1002/hbm.22017

Page 16/25 
15. Catani, M, M P Allin, M Husain, L Pugliese, M M Mesulam, R M Murray and D K Jones (2007) Symmetries in human brain language pathways correlate with verbal recall. Proc Natl Acad Sci U S A 43, 17163-8 doi:

$10.1073 /$ pnas.0702116104

16. Catani, M, D K Jones and D H ffytche (2005) Perisylvian language networks of the human brain. Ann Neuro/ 1, 8-16 doi: 10.1002/ana.20319

17. Catani, $M$ and $M$ Thiebaut de Schotten (2008) A diffusion tensor imaging tractography atlas for virtual in vivo dissections. Cortex 8, 1105-32 doi: 10.1016/j.cortex.2008.05.004

18. Chen, X, R C Anderson, W Li, M Hao, X Wu and H Shu (2004) Phonological Awareness of Bilingual and Monolingual Chinese Children. Journal of Educational Psychology 1, 142-151 doi: 10.1037/0022-0663.96.1.142

19. Connally, E L, D Ward, P Howell and K E Watkins (2014) Disrupted white matter in language and motor tracts in developmental stuttering. Brain Lang 25-35 doi: 10.1016/j.bandl.2013.05.013 List of multilingual countries and regions. https://en.wikipedia.org/w/index.php?title=List_of_multilingual_countries_and_regions\&oldid=1008121326

20. Costa, A and N Sebastian-Galles (2014) How does the bilingual experience sculpt the brain? Nat Rev Neurosci 5, 33645 doi: $10.1038 /$ nrn3709

21. Cummine, $J$ and $C A$ Boliek (2013) Understanding white matter integrity stability for bilinguals on language status and reading performance. Brain Struct Funct 2, 595-601 doi: 10.1007/s00429-012-0466-6

22. D'Ausilio, A, J Jarmolowska, P Busan, I Bufalari and L Craighero (2011) Tongue corticospinal modulation during attended verbal stimuli: priming and coarticulation effects. Neuropsychologia 13, 3670-6 doi:

10.1016/j.neuropsychologia.2011.09.022

23. De Schotten, M T, F Dell'Acqua, S Forkel, A Simmons, F Vergani, D G Murphy and M Catani (2011) A lateralized brain network for visuo-spatial attention. Nature Precedings 1-1 doi,

24. Deng, Y, Q Wu and X Weng (2013) Unimodal and multimodal regions for logographic language processing in left ventral occipitotemporal cortex. Front Hum Neurosci 619 doi: 10.3389/fnhum.2013.00619

25. Douglas, R (2008) White Matter Matters. Scientific American 3, 54-61

26. Duffau, H (2015) Stimulation mapping of white matter tracts to study brain functional connectivity. Nat Rev Neurol 5, 255-65 doi: 10.1038/nrneurol.2015.51

27. Fan, X, Y Wu, L Cai, J Ma, N Pan, X Xu, T Sun, J Jing and X Li (2021) The Differences in the Whole-Brain Functional Network between Cantonese-Mandarin Bilinguals and Mandarin Monolinguals. Brain Sci 3, doi:

10.3390/brainsci11030310

28. Farinella-Bocian, K, E Strand and E Benarroch (2007) Neuroanatomical review of mechanisms important to speech motor control. Perspectives on Neurophysiology and Neurogenic Speech and Language Disorders 2, 8-12 doi,

29. Fields, R D (2015) A new mechanism of nervous system plasticity: activity-dependent myelination. Nat Rev Neurosci 12, 756-67 doi: 10.1038/nrn4023

30. Friesen, D C, D Jared and C A Haigh (2014) Phonological processing dynamics in bilingual word naming. Can J Exp Psycho/3, 179-93 doi: 10.1037/cep0000026

31. Giacosa, C, F J Karpati, N E V Foster, K L Hyde and V B Penhune (2019) The descending motor tracts are different in dancers and musicians. Brain Struct Funct 9, 3229-3246 doi: 10.1007/s00429-019-01963-0

32. Gibson, E M, D Purger, C W Mount, A K Goldstein, G L Lin, L S Wood, I Inema, S E Miller, G Bieri, J B Zuchero, B A Barres, P J Woo, H Vogel and M Monje (2014) Neuronal activity promotes oligodendrogenesis and adaptive myelination in the mammalian brain. Science 6183, 1252304 doi: 10.1126/science. 1252304

33. Gold, B T, N F Johnson and D K Powell (2013) Lifelong bilingualism contributes to cognitive reserve against white matter integrity declines in aging. Neuropsychologia 13, 2841-6 doi: 10.1016/j.neuropsychologia.2013.09.037 
34. Goldstein, A, B P Covington, N Mahabadi and F B Mesfin (2020) Neuroanatomy, Corpus Callosum. Treasure Island $(\mathrm{FL})$ : pp.

35. Green, D W and J Abutalebi (2013) Language control in bilinguals: The adaptive control hypothesis. Journal of Cognitive Psychology 5, 515-530 doi: 10.1080/20445911.2013.796377

36. Grundy, J G and K Timmer (2016) Bilingualism and working memory capacity: A comprehensive meta-analysis. Second Language Research 3, 325-340 doi: 10.1177/0267658316678286

37. Hamalainen, S, V Sairanen, A Leminen and M Lehtonen (2017) Bilingualism modulates the white matter structure of language-related pathways. Neuroimage 249-257 doi: 10.1016/j.neuroimage.2017.02.081

38. Herbet, G, I Zemmoura and H Duffau (2018) Functional Anatomy of the Inferior Longitudinal Fasciculus: From Historical Reports to Current Hypotheses. Front Neuroanat 77 doi: 10.3389/fnana.2018.00077

39. Hickok, G and D Poeppel (2007) The cortical organization of speech processing. Nat Rev Neurosci 5, 393-402 doi: $10.1038 / \mathrm{nrn} 2113$

40. Hosoda, C, K Tanaka, T Nariai, M Honda and T Hanakawa (2013) Dynamic neural network reorganization associated with second language vocabulary acquisition: a multimodal imaging study. J Neurosci 34, 13663-72 doi: 10.1523/jneurosci.0410-13.2013

41. Hua, K, J Zhang, S Wakana, H Jiang, X Li, D S Reich, P A Calabresi, J J Pekar, P C van Zijl and S Mori (2008) Tract probability maps in stereotaxic spaces: analyses of white matter anatomy and tract-specific quantification. Neuroimage 1, 336-47 doi: 10.1016/j.neuroimage.2007.07.053

42. Huiming, $X$ and $Z$ Zhe (2016) On Language Use and Language Attitude of the Youths and Adolescents in Guangzhou. Applied Linguistic 03, 20-29 doi,

43. Hull, R and J Vaid (2007) Bilingual language lateralization: a meta-analytic tale of two hemispheres. Neuropsychologia 9, 1987-2008 doi: 10.1016/j.neuropsychologia.2007.03.002

44. Jang, S H and S S Yeo (2014) Thalamocortical connections between the mediodorsal nucleus of the thalamus and prefrontal cortex in the human brain: a diffusion tensor tractographic study. Yonsei Med J 3, 709-14 doi: 10.3349/ymj.2014.55.3.709

45. Jian, H, L Aitao and Z Jijia (2011) Cross-language Interference in the Verbal Fluency of Mandarin-Cantanese Diglossia People [J]. Psychological Research doi,

46. Jin, T and S G Kim (2008) Functional changes of apparent diffusion coefficient during visual stimulation investigated by diffusion-weighted gradient-echo fMRI. Neuroimage 3, 801-12 doi:

10.1016/j.neuroimage.2008.03.014

47. Just, M A, V L Cherkassky, T A Keller, R K Kana and N J Minshew (2007) Functional and anatomical cortical underconnectivity in autism: evidence from an FMRI study of an executive function task and corpus callosum morphometry. Cereb Cortex 4, 951-61 doi: 10.1093/cercor/bhl006

48. Kim, S Y, T Qi, X Feng, G Ding, L Liu and F Cao (2016) How does language distance between L1 and L2 affect the L2 brain network? An fMRI study of Korean-Chinese-English trilinguals. Neuroimage 25-39 doi:

10.1016/j.neuroimage.2015.11.068

49. Kroll, J F, S C Bobb and N Hoshino (2014) Two languages in mind: Bilingualism as a tool to investigate language, cognition, and the brain. Curr Dir Psychol Sci 3, 159-163 doi: 10.1177/0963721414528511

50. Kroll, J F, P E Dussias, K Bice and L Perrotti (2015) Bilingualism, Mind, and Brain. Annual Review of Linguistics 1, 377-394 doi: 10.1146/annurev-linguist-030514-124937

51. Kuhl, P K, J Stevenson, N M Corrigan, J J F van den Bosch, D D Can and T Richards (2016) Neuroimaging of the bilingual brain: Structural brain correlates of listening and speaking in a second language. Brain Lang 1-9 doi: 10.1016/j.bandl.2016.07.004

Page $18 / 25$ 
52. Lebel, C, B Shaywitz, J Holahan, S Shaywitz, K Marchione and C Beaulieu (2013) Diffusion tensor imaging correlates of reading ability in dysfluent and non-impaired readers. Brain Lang 2, 215-22 doi: 10.1016/j.bandl.2012.10.009

53. Li, J (1990) Cantonese is independent of Chinese.pp. 54-76.

54. Li, M, Y Zhang, L Song, R Huang, J Ding, Y Fang, Y Xu and Z Han (2017) Structural connectivity subserving verbal fluency revealed by lesion-behavior mapping in stroke patients. Neuropsychologia 85-96 doi: 10.1016/j.neuropsychologia.2017.05.008

55. Li, P, J Legault and K A Litcofsky (2014) Neuroplasticity as a function of second language learning: anatomical changes in the human brain. Cortex 301-24 doi: 10.1016/j.cortex.2014.05.001

56. Li, X, D Yang, J Jing, J Zheng, D Luo, X Wang and X Zhang (2011) Mandarin phonological process in mandarinspoken university students and Cantonese-spoken university students. Chinese Mental Health Journal 7, 528-532 doi,

57. Liang, B and Y Du (2018) The Functional Neuroanatomy of Lexical Tone Perception: An Activation Likelihood Estimation Meta-Analysis. Front Neurosci 495 doi: 10.3389/fnins.2018.00495

58. Liu, H and F Cao (2016) L1 and L2 processing in the bilingual brain: A meta-analysis of neuroimaging studies. Brain Lang 60-73 doi: 10.1016/j.bandl.2016.05.013

59. Luk, G, E Bialystok, F I Craik and C L Grady (2011) Lifelong bilingualism maintains white matter integrity in older adults. J Neurosci 46, 16808-13 doi: 10.1523/jneurosci.4563-11.2011

60. Ma, J, Y Wu, T Sun, L Cai, X Fan and X Li (2020) Neural substrates of bilingual processing in a logographic writing system: An fMRI study in Chinese Cantonese-Mandarin bilinguals. Brain Res 146794 doi:

10.1016/j.brainres.2020.146794

61. Mamiya, P C, T L Richards, B P Coe, E E Eichler and P K Kuhl (2016) Brain white matter structure and COMT gene are linked to second-language learning in adults. Proc Natl Acad Sci U S A 26, 7249-54 doi: 10.1073/pnas.1606602113

62. Mamiya, P C, T L Richards and P K Kuhl (2018) Right Forceps Minor and Anterior Thalamic Radiation Predict Executive Function Skills in Young Bilingual Adults. Front Psychol 118 doi: 10.3389/fpsyg.2018.00118

63. Marian, V and A Shook (2012) The cognitive benefits of being bilingual.Dana Foundation, pp.

64. McKenzie, I A, D Ohayon, H Li, J P de Faria, B Emery, K Tohyama and W D Richardson (2014) Motor skill learning requires active central myelination. Science 6207, 318-22 doi: 10.1126/science.1254960

65. McPherson, W B, P T Ackerman and R A Dykman (1997) Auditory and visual rhyme judgements reveal differences and similarities between normal and disabled adolescent readers. Dyslexia 2, 63-77 doi,

66. Mei, L, G Xue, Z L Lu, Q He, M Wei, M Zhang, Q Dong and C Chen (2015) Native language experience shapes neural basis of addressed and assembled phonologies. Neuroimage 38-48 doi: 10.1016/j.neuroimage.2015.03.075

67. Mirman, D, Q Chen, Y Zhang, Z Wang, O K Faseyitan, H B Coslett and M F Schwartz (2015) Neural organization of spoken language revealed by lesion-symptom mapping. Nat Commun 6762 doi: 10.1038/ncomms7762

68. Nakajima, R, M Kinoshita, H Shinohara and M Nakada (2019) The superior longitudinal fascicle: reconsidering the fronto-parietal neural network based on anatomy and function. Brain Imaging Behav doi: 10.1007/s11682-01900187-4

69. Singh, N, Rajan, A; Malagi, A, Ramanujan K, Canini M, Della Rosa, P, Raghunathan, P, Weekes, B (2017) Microstructural anatomical differences between bilinguals and monolinguals. Bilingualism: Language and Cognition 5, 14 doi: 10.1017/S1366728917000438

70. Nosarti, C, A Mechelli, D W Green and C J Price (2010) The impact of second language learning on semantic and nonsemantic first language reading. Cereb Cortex 2, 315-27 doi: 10.1093/cercor/bhp101

71. Oldfield, R C (1971) The assessment and analysis of handedness: the Edinburgh inventory. Neuropsychologia 1, 97113 doi, 
72. Parker Jones, O, D W Green, A Grogan, C Pliatsikas, K Filippopolitis, N Ali, H L Lee, S Ramsden, K Gazarian, S Prejawa, M L Seghier and C J Price (2012) Where, when and why brain activation differs for bilinguals and monolinguals during picture naming and reading aloud. Cereb Cortex 4, 892-902 doi: 10.1093/cercor/bhr161

73. Pennino, A M (2010) Monolingual and Bilingual Spanish-English Children's Phonological Production on Rapid Automatized Naming Tasks.University of Memphis, pp.

74. Perez-Iglesias, R, D Tordesillas-Gutierrez, P K McGuire, G J Barker, R Roiz-Santianez, I Mata, E M de Lucas, J M Rodriguez-Sanchez, R Ayesa-Arriola, J L Vazquez-Barquero and B Crespo-Facorro (2010) White matter integrity and cognitive impairment in first-episode psychosis. Am J Psychiatry 4, 451-8 doi: 10.1176/appi.ajp.2009.09050716

75. Pliatsikas, C, L Meteyard, J Veríssimo, V DeLuca, K Shattuck and M T Ullman (2020) The effect of bilingualism on brain development from early childhood to young adulthood. Brain Struct Funct 7, 2131-2152 doi: 10.1007/s00429020-02115-5

76. Pliatsikas, C, E Moschopoulou and J D Saddy (2015) The effects of bilingualism on the white matter structure of the brain. Proc Natl Acad Sci U S A 5, 1334-7 doi: 10.1073/pnas.1414183112

77. Qi, Z, M Han, K Garel, E San Chen and J D E Gabrieli (2015) White-matter structure in the right hemisphere predicts Mandarin Chinese learning success. Journal of Neurolinguistics 14-28 doi: https://doi.org/10.1016/j.jneuroling.2014.08.004

78. Rossi, E, H Cheng, J F Kroll, M T Diaz and S D Newman (2017) Changes in White-Matter Connectivity in Late Second Language Learners: Evidence from Diffusion Tensor Imaging. Front Psycho/ 2040 doi: 10.3389/fpsyg.2017.02040

79. Sagi, Y, I Tavor, S Hofstetter, S Tzur-Moryosef, T Blumenfeld-Katzir and Y Assaf (2012) Learning in the fast lane: new insights into neuroplasticity. Neuron 6, 1195-203 doi: 10.1016/j.neuron.2012.01.025

80. Sarubbo, S, A De Benedictis, S Merler, E Mandonnet, M Barbareschi, M Dallabona, F Chioffi and H Duffau (2016) Structural and functional integration between dorsal and ventral language streams as revealed by blunt dissection and direct electrical stimulation. Hum Brain Mapp 11, 3858-3872 doi: 10.1002/hbm.23281

81. Schlaggar, B L and B D McCandliss (2007) Development of neural systems for reading. Annu Rev Neurosci $475-503$ doi: 10.1146/annurev.neuro.28.061604.135645

82. Schlegel, A A, J J Rudelson and P U Tse (2012) White matter structure changes as adults learn a second language. $J$ Cogn Neurosci 8, 1664-70 doi: 10.1162/jocn_a_00240

83. Scholz, J, M C Klein, T E Behrens and H Johansen-Berg (2009) Training induces changes in white-matter architecture. Nat Neurosci 11, 1370-1 doi: 10.1038/nn.2412

84. Shin, J, J Rowley, R Chowdhury, P Jolicoeur, D Klein, C Grova, P Rosa-Neto and E Kobayashi (2019) Inferior Longitudinal Fasciculus' Role in Visual Processing and Language Comprehension: A Combined MEG-DTI Study. Front Neurosci 875 doi: 10.3389/fnins.2019.00875

85. Shu, H, H Peng and C McBride-Chang (2008) Phonological awareness in young Chinese children. Dev Sci 1, 171-81 doi: 10.1111/j.1467-7687.2007.00654.x

86. Siddaiah, A, M Saldanha, S K Venkatesh, N B Ramachandra and P Padakannaya (2016) Development of Rapid Automatized Naming (RAN) in Simultaneous Kannada-English Biliterate Children. J Psycholinguist Res 1, 177-87 doi: 10.1007/s10936-014-9338-y

87. Smith, S M (2002) Fast robust automated brain extraction. Hum Brain Mapp 3, 143-55 doi: 10.1002/hbm.10062

88. Smith, S M, M Jenkinson, H Johansen-Berg, D Rueckert, T E Nichols, C E Mackay, K E Watkins, O Ciccarelli, M Z Cader, P M Matthews and T E J Behrens (2006) Tract-based spatial statistics: Voxelwise analysis of multi-subject diffusion data. Neuroimage 4, 1487-1505 doi: 10.1016/j.neuroimage.2006.02.024

89. Smith, S M and T E Nichols (2009) Threshold-free cluster enhancement: addressing problems of smoothing, threshold dependence and localisation in cluster inference. Neuroimage 1, 83-98 doi: 
10.1016/j.neuroimage.2008.03.061

90. Smolker, H R, N P Friedman, J K Hewitt and M T Banich (2018) Neuroanatomical Correlates of the Unity and Diversity Model of Executive Function in Young Adults. Front Hum Neurosci 283 doi: 10.3389/fnhum.2018.00283

91. Soares, J, P Marques, V Alves and N Sousa (2013) A hitchhiker's guide to diffusion tensor imaging. Frontiers in neuroscience 31 doi,

92. Solowij, N, A Zalesky, V Lorenzetti and M Yücel (2017) Chronic Cannabis Use and Axonal Fiber Connectivity.Elsevier, pp. 391-400.

93. Song, S K, S W Sun, M J Ramsbottom, C Chang, J Russell and A H Cross (2002) Dysmyelination revealed through MRI as increased radial (but unchanged axial) diffusion of water. Neuroimage 3, 1429-36 doi:

10.1006/nimg.2002.1267

94. Stein, M, C Winkler, A Kaiser and T Dierks (2014) Structural brain changes related to bilingualism: does immersion make a difference? Front Psychol 1116 doi: 10.3389/fpsyg.2014.01116

95. Su, M, J Zhao, M Thiebaut de Schotten, W Zhou, G Gong, F Ramus and H Shu (2018) Alterations in white matter pathways underlying phonological and morphological processing in Chinese developmental dyslexia. Dev Cogn Neurosci 11-19 doi: 10.1016/j.dcn.2018.04.002

96. Sulpizio, S, N Del Maschio, D Fedeli and J Abutalebi (2020) Bilingual language processing: A meta-analysis of functional neuroimaging studies. Neurosci Biobehav Rev 834-853 doi: 10.1016/j.neubiorev.2019.12.014

97. Sun, Y, Y Yang, A S Desroches, L Liu and D Peng (2011) The role of the ventral and dorsal pathways in reading Chinese characters and English words. Brain Lang 2, 80-8 doi: 10.1016/j.bandl.2011.03.012

98. Tan, L H, A R Laird, K Li and P T Fox (2005) Neuroanatomical correlates of phonological processing of Chinese characters and alphabetic words: a meta-analysis. Hum Brain Mapp 1, 83-91 doi: 10.1002/hbm.20134

99. Tardif, T, P Fletcher, W Liang and N Kaciroti (2009) Early vocabulary development in Mandarin (Putonghua) and Cantonese. J Child Lang 5, 1115-44 doi: 10.1017/S0305000908009185

100. TÊ WANG, Y (1960) The Lexicostatistic Estimation of the Time Depths of the Five Main Chinese Dialects. GENGO KENKYU (Journal of the Linguistic Society of Japan) 38, 33-105 doi,

101. Tomasino, B, T lus, M Skrap and C Luzzatti (2020) Phonological and surface dyslexia in individuals with brain tumors: Performance pre-, intra-, immediately post-surgery and at follow-up. Hum Brain Mapp 17, 5015-5031 doi: 10.1002/hbm. 25176

102. Vaessen, M J, A Saj, K O Lovblad, M Gschwind and P Vuilleumier (2016) Structural white-matter connections mediating distinct behavioral components of spatial neglect in right brain-damaged patients. Cortex 54-68 doi: 10.1016/j.cortex.2015.12.008

103. Vanderauwera, J, J Wouters, M Vandermosten and P Ghesquiere (2017) Early dynamics of white matter deficits in children developing dyslexia. Dev Cogn Neurosci 69-77 doi: 10.1016/j.dcn.2017.08.003

104. Vandermosten, M, B Boets, J Wouters and P Ghesquiere (2012) A qualitative and quantitative review of diffusion tensor imaging studies in reading and dyslexia. Neurosci Biobehav Rev 6, 1532-52 doi:

10.1016/j.neubiorev.2012.04.002

105. Vassal, M, E Le Bars, S Moritz-Gasser, N Menjot and H Duffau (2010) Crossed aphasia elicited by intraoperative cortical and subcortical stimulation in awake patients. J Neurosurg 6, 1251-8 doi: 10.3171/2010.6.JNS10719

106. Wagner, R K and J K Torgesen (1987) The nature of phonological processing and its causal role in the acquisition of reading skills. Psychol Bull 2, 192-212 doi: 10.1037/0033-2909.101.2.192

107. Wang, K, X Li, R Huang, J Ding, L Song and Z Han (2020) The left inferior longitudinal fasciculus supports orthographic processing: Evidence from a lesion-behavior mapping analysis. Brain Lang 104721 doi: https://doi.org/10.1016/j.bandl.2019.104721

Page 21/25 
108. Welniarz, Q, I Dusart and E Roze (2017) The corticospinal tract: Evolution, development, and human disorders. Dev Neurobiol 7, 810-829 doi: 10.1002/dneu.22455

109. Winkler, A M, G R Ridgway, M A Webster, S M Smith and T E Nichols (2014) Permutation inference for the general linear model. Neuroimage 381-97 doi: 10.1016/j.neuroimage.2014.01.060

110. Yendiki, A, K Koldewyn, S Kakunoori, N Kanwisher and B Fischl (2014) Spurious group differences due to head motion in a diffusion MRI study. Neuroimage 79-90 doi: 10.1016/j.neuroimage.2013.11.027

111. Yeung, S S (2016) Cognitive Mechanism Underlying the Relationship Between Rapid Automatized Naming and Reading: A Longitudinal Study on Bilingual Children. Reading Psychology 8, 1196-1211 doi,

112. Zhang, M, C Chen and G Xue (2014) Language-general and -specific white matter microstructural bases for reading. Neuroimage 7 doi: 10.1016/j.neuroimage.2014.04.080

\section{Figures}




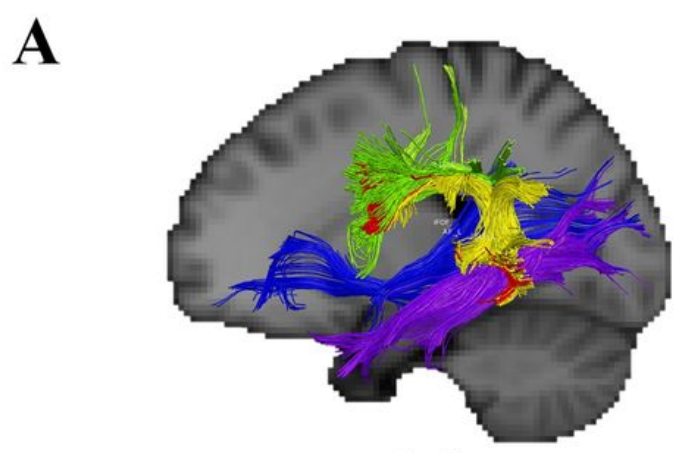

Left

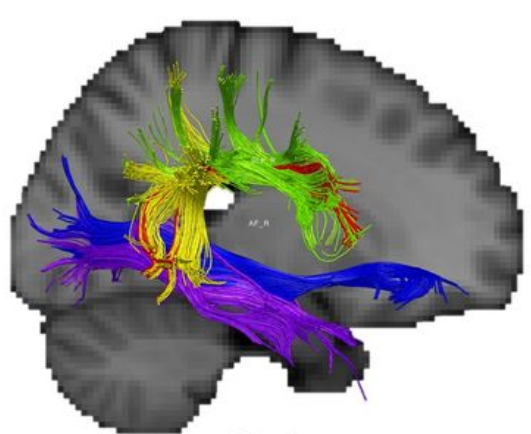

Right

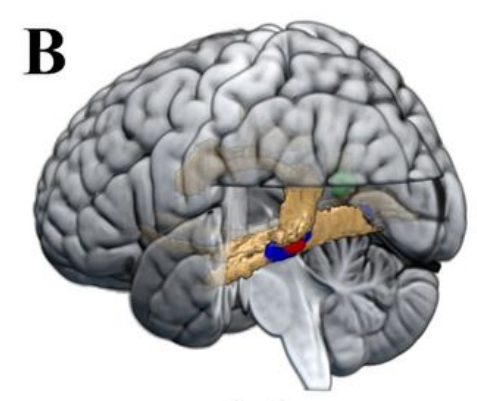

Left

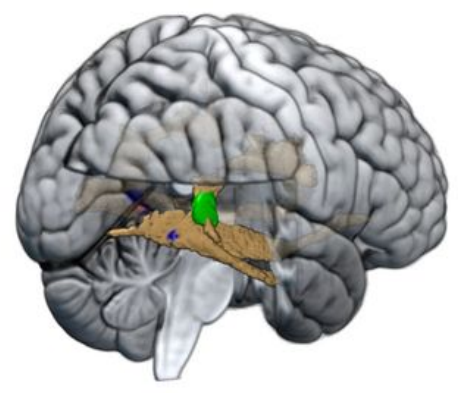

Right

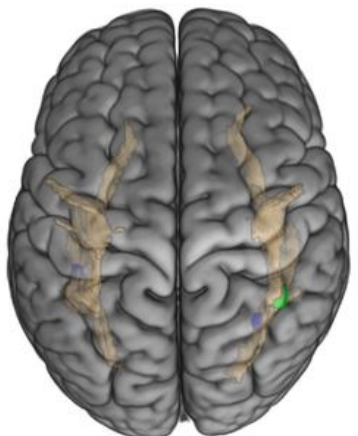

Superior

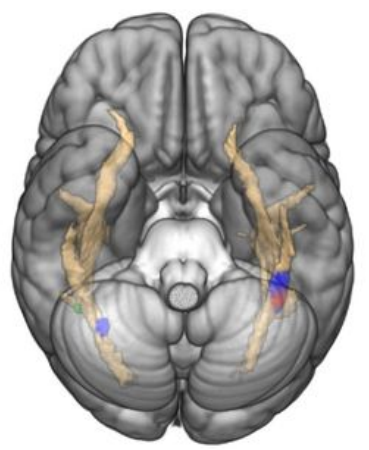

Inferior

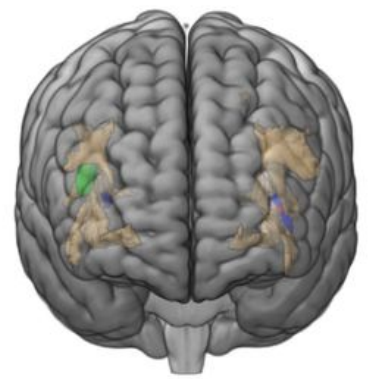

Anterior

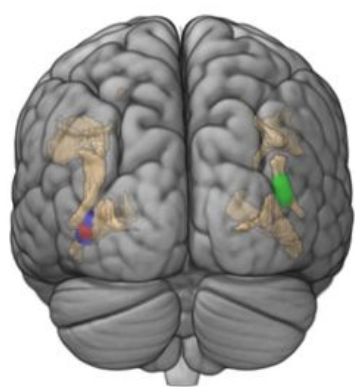

Posterior

Figure 1

Significant anatomical structures in the tract-specific TBSS analysis. (A) The five tracts of interest (TOls): fSLF (green), AF (red), tSLF (yellow), ILF (purple), IFOF (blue) overlaid on a standard MNI brain. (B) The significant clusters in tractspecific TBSS analysis. The binary mask of tracts of interest is copper while the significant clusters in the comparison of $F A, M D$ and $A D$ is in red (FA), green (MD) and blue (AD) respectively. 


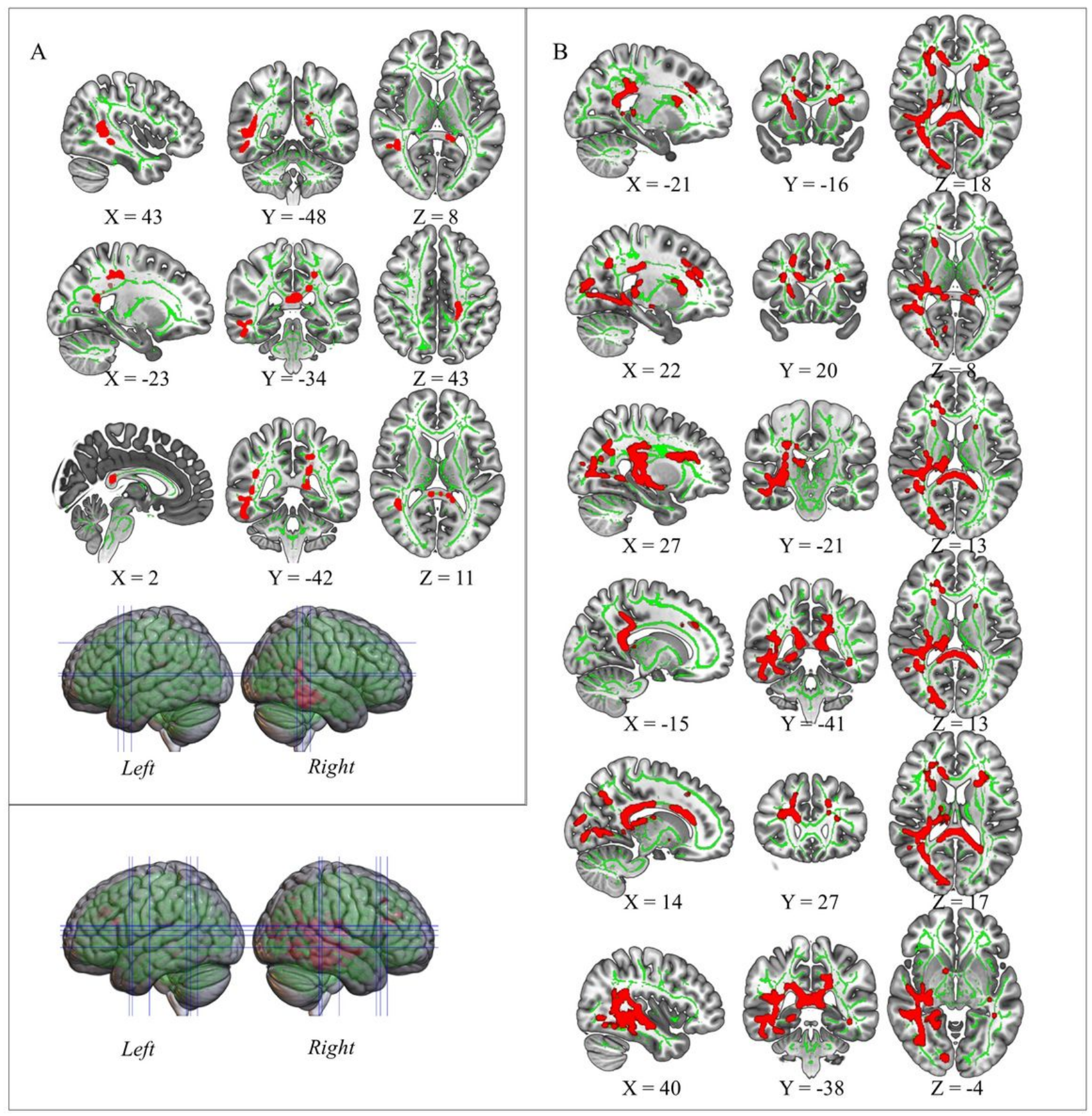

Figure 2

The significant clusters in the whole-brain TBSS analysis. (A) The significant clusters in the whole-brain-level comparison of MD. (B) The significant clusters in the whole-brain-level comparison of AD. Mean FA skeleton is green and significant clusters emphasized via tbss-fill is red. 

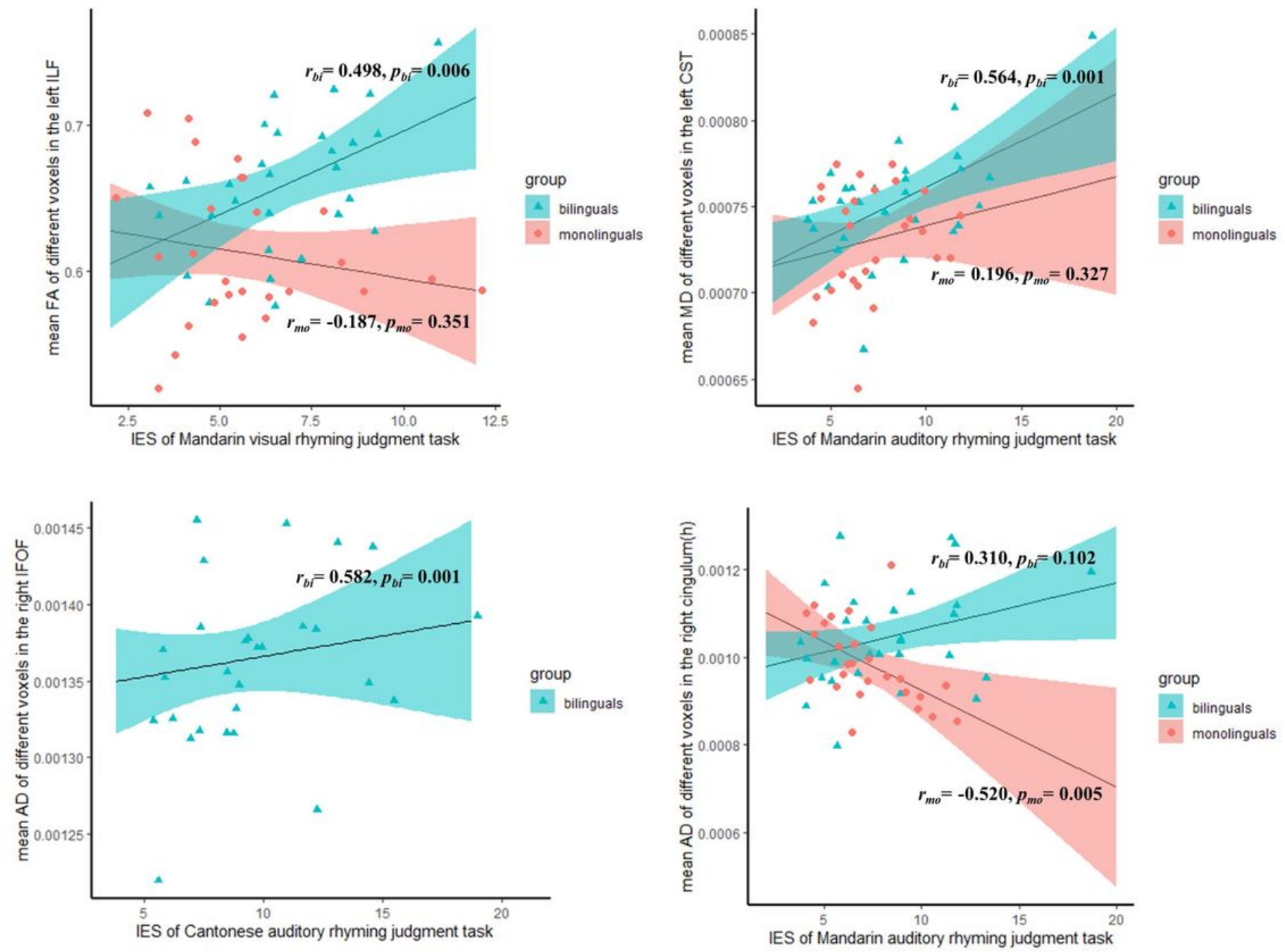

\section{Figure 3}

The significant correlation between the mean different DTI indices of significant voxels in the tracts and behavioral measures, within the subgroups.

\section{Supplementary Files}

This is a list of supplementary files associated with this preprint. Click to download.

- SupplementalFigure1.docx

- SupplementalTable1.docx 